\title{
Surface Quality Improvement of Atmospheric Pressure Plasma Polishing (APPP) in Machining of Silicon Ultra-Smooth Surfaces*
}

\author{
Jufan ZHANG**, Bing LI**, Bo WANG*** and Shen DONG*** \\ ** Shenzhen Graduate School, Harbin Institute of Technology \\ HIT Campus of Shenzhen University Town, Xili, Shenzhen, China \\ E-mail: zhangjufan@hitsz.edu,cn \\ *** School of Mechanical Engineering, Harbin Institute of Technology \\ 92 Xi Da Zhi Street, Harbin, China
}

\begin{abstract}
To produce ultra-smooth optical surfaces on hard brittle materials and functional crystal materials without surface damage, atmospheric pressure plasma polishing (APPP) is developed. And improvement of surface quality by APPP process is analyzed by theoretical simulation and experiments. Quantum chemistry simulation proves that convex topography is removed faster than concave topography, thus surface roughness can be reduced further to form ultra-smooth surface. Experiment on single crystal silicon wafer also shows the surface roughness decreases from Ra $1.179 \mathrm{~nm}$ to Ra $0.483 \mathrm{~nm}$. AFM (atomic force microscope) measurement results of specified roughness index taken at different moment in machining process accord well with theoretical simulation. Average reduced-modulus measured by nano-mechanical test system rises from 14.65GPa to 36.10GPa which demonstrates the improvement of surface mechanical properties. Further calculation also indicates the average residual stress is reduced by $7.64 \mathrm{GPa}$ after machining which reflects the elimination of former deformation layer. Element analysis by X-ray photoelectron spectroscopy and X-ray diffraction indicates that chemical composition is also purified without new contamination introduced, and the overall status of machined surface goes closer to ideal silicon surface with better performance.
\end{abstract}

Key words: Atmospheric Pressure Plasma, Polishing, Single Crystal Silicon, Surface Quality, Ultra-Smooth Surface

\section{Introduction}

Development of modern optical industry demands more and more perfect surface performance and presents a number of stringent challenges to optics manufacturing technologies. The desired high quality optical surfaces not only require extremely low surface roughness at nanometer level in order to achieve high reflectivity, but also strive for minimum surface and subsurface damage to obtain expected performance and prolong service life ${ }^{(1)}$. Especially recent years, with utilization of SiC, KDP and many other hard brittle materials and functional crystal materials in the defense, aerospace and civil fields, conventional optics manufacturing technologies can't support accomplishing so rigorous machining processing for such high quality optical surfaces. And some new technologies developed in the last decades, such as float polishing, plasma assisted chemical etching, ion beam polishing, magnetorheological polishing and so on, have achieved nearly perfect

Received 10 Feb., 2012 (No. 12-0109) [DOI: 10.1299/jamdsm.6.464]

Copyright () 2012 by JSME 
surfaces on some materials without any surface defect. However, subsurface damage concealed below can not be avoided yet as the inevitable consequence of force exertion, which influences surface performance, maintenance cost and service life significantly ${ }^{(2)(3)}$. Occasionally, outside instantaneous stress applied on surface unexpectedly even leads directly to component scrap and causes disastrous consequence ${ }^{(4)}$. And subsurface damage has been thought to be reduced by several rounds of grinding and polishing with smaller and smaller grit size, along with adequate removal depth. Although this method maybe works to prevent introduction of new damage, the machined surface is still testified to be under substantial residual stresses ${ }^{(5)}$. And the processing efficiency is usually too low to support industrial manufacturing. Moreover, for high hardness material, like $\mathrm{SiC}$ which is extremely attractive for reflective optics of large ground and space-based telescope, the diamond grinding tools are always required to achieve effective shaping and polishing. But the high downward pressure demanded to use these tools inevitably produces substantial sub-surface damage and stress. And $\mathrm{SiC}$ is chemically inert to acid solvent at normal condition so there is no effective means to easily reduce subsurface damage, which makes it a difficult issue to obtain totally satisfied quality on SiC optical surface. Aiming at these problems, much effort is devoted to developing new non-contact machining methods which bring in completely innovative mechanism and facility to accomplish material removal by chemical process to avoid surface damage caused by mechanical forces ${ }^{(6)}$. Atmospheric Pressure Plasma Polishing (APPP) is a kind of such technology, which depends on the chemical reaction between reactive plasma and workpiece surface to produce high quality optical surfaces with minimum defects ${ }^{(7)(8)}$. The chemical nature is helpful to improve optical surface's properties, which is studied in this paper both in theoretic simulation and experiments.

\section{Principle and Facility of APPP}

Atmospheric pressure plasma polishing (APPP) utilizes atmospheric pressure plasma to excite reaction gas to generate reactive radicals. Then reactive radicals react chemically with the surface atoms to accomplish atom-scale material removal. The products are always volatile gases that tend to vent to avoid introducing new surface contamination. For example, to machine single crystal silicon wafer, helium $(\mathrm{He})$ gas is usually selected as plasma gas while carbon tetrafluoride $\left(\mathrm{CF}_{4}\right)$ gas works as the reactive precursor. $\mathrm{CF}_{4}$ molecules are dissociated due to the excitation of plasma and release radical fluorine atoms which are high chemically reactive. The radical fluorine atoms react with surface silicon atoms producing $\mathrm{SF}_{4}$ gas product, as Eq.(1) and (2) shows. Fig.1 illustrates the general process of the chemical reaction occurring on the surface.

$$
\begin{aligned}
& \mathrm{CF}_{4}+e^{-*} \rightarrow \mathrm{CF}_{3} \cdot+\mathrm{F} \cdot+e^{-} \\
& \mathrm{Si}+4 \mathrm{~F} \cdot \rightarrow \mathrm{SiF}_{4}
\end{aligned}
$$
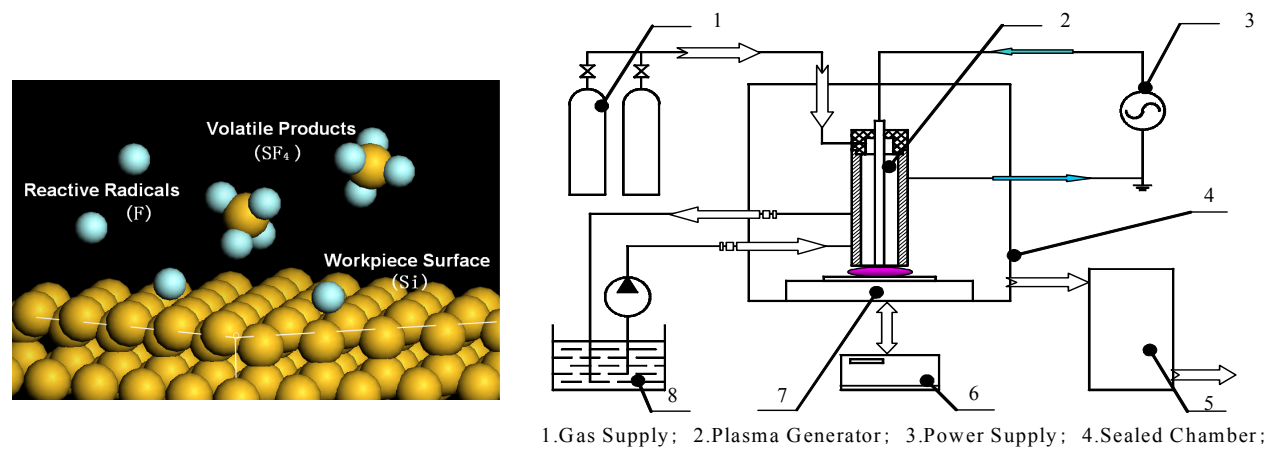

Fig.1 Principle of atmospheric pressure plasma polishing

Fig. 2 Schematic diagram of APPP system 
The facility supporting APPP is illustrated as Fig.2, which contains a radio frequency capacitively coupled plasma torch as core component ${ }^{(8)}$.

\section{Improvement of Surface Topography}

One of the most important indices to evaluate machining effect is surface roughness. Always there are still some microscopic uneven structures on optical surfaces which can be weakened by ultra-precision finishing step, so surface topography is improved to form ultra-smooth surface. Since APPP is chemical in nature, classical Newtonian mechanics is not suitable to be used to analyze the mechanism. And for studying actions between individual atoms, quantum chemistry theory is justified a very useful tool and some commercial software is available for simulation. Thus surface topography improvement of APPP is studied both by quantum chemistry simulation and experiments.

\subsection{Quantum Chemistry Simulation}

Preliminary simulation mainly focuses on the diversity of removal rate of different surface topographies in chemical reaction, which is also the foundation to reveal formation mechanism of ultra-smooth surface in APPP further. And considerate simplification is necessary to design appropriate models, since actual surface topography is too complex which is impossible to be entirely duplicated. Here, "appropriate models" means the models should meet following two rules at least: (1) reflecting the desired characteristics of demanded surface structures, (2) suitable for computation with computer. Thus, according to the principle intent, surface topography is generally divided into two sorts by surface plane, convex structure above surface and concave structure below surface. Provided APPP process indicates selectivity to different topographies, the surface roughness can be changed. For more details, from the general sense, convex topography should be removed faster than concave topography, as result the whole surface could gradually go flattened. Contrarily, if convex topography reacts more slowly than concave topography, topography difference will become more distinct. Of course, actual surface status is more complex, maybe there is not a plane existing as division interface, maybe each surface structure contains many various sub-structures. But note that as the final finishing step, APPP is always used on optical surfaces which have already been under smooth status with low roughness and relatively regular topography, and this simulation mainly aims at proving the roughness decreasing of APPP in theory, so the simplification is reasonable, which is also testified by experiments later.

Simulation is completed by CASTEP module of Material Studio software (4.0, Accelrys Software Inc.). Consistent with machining experiments with $\mathrm{He} / \mathrm{CF}_{4}$ gas recipe on single crystal silicon wafer, simulation mainly focuses on interaction between silicon( $\mathrm{Si}$ ) atoms and fluorine(F) atoms. Many typical parameters are calculated in simulation, some of which can even be illustrated by visual models, but here just system energy is discussed to calculate binding energy further. Binding energy is defined as the difference of system energy before and after reaction, which reflects the possibility of certain chemical reaction. If the value is minus, usually it means the reaction releases energy and the system becomes more stable. Generally, the bigger the absolute value is, the more possible the chemical reaction occurs.

Chemical reaction between $\mathrm{Si}$ atoms and $\mathrm{F}$ atoms can be regarded as the bonding process of radical $\mathrm{F}$ atoms with surface $\mathrm{Si}$ atoms. Thus, the model of original $\mathrm{Si}(100)$ surface before reaction can be built firstly by cutting silicon crystal cell along (100) direction with depth of two layers. Then super cell contains $2 \times 2$ original cells at two dimensions with $10 \AA$ vacuum layer depth. Based on this periodic structure with reasonable body configuration, by removing certain atoms according to some rules, convex and concave structures can be shaped. Both models are also need to be optimized because 
surface lattice will change obviously with absence of some atoms. But limited by computer capacity, atom amounts of both models are assigned nearly 60, as shown in Fig.3.

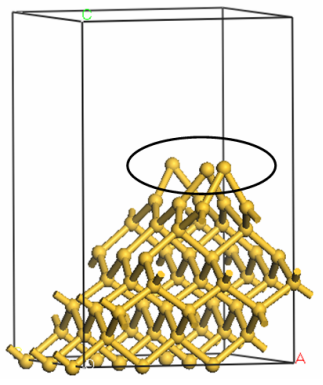

(a) Model of convex structure

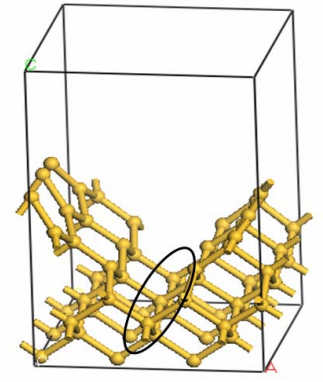

(b) Model of concave structure

Fig. 3 Models of convex and concave structures on $\mathrm{Si}(100)$ surface

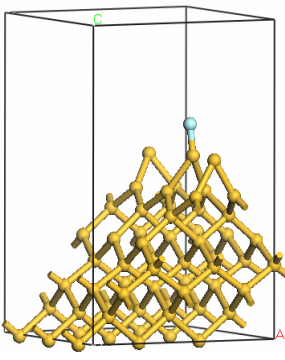

(a) Model of $\mathrm{Si}(100)$ peak with bonding(b)
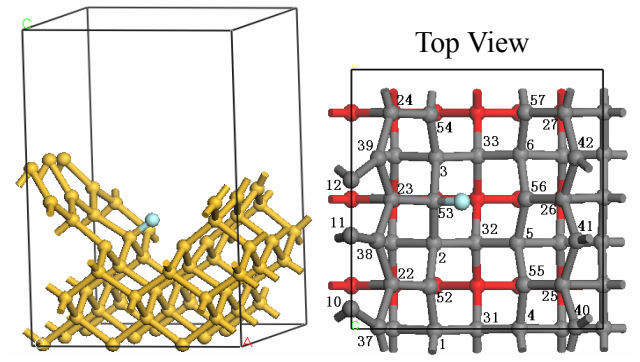

(b) Model of $\mathrm{Si}(100)$ valley with bonding

Fig. 4 Models of the typical $\mathrm{Si}(100)$ surface structures with bonding

By selecting most representative positions for Si-F bonding, models after reaction can be built as Fig.4 shows. Here, the lowest position in concave model and highest position in convex model are originally thought to be most representative, as surrounded by cycles in Fig.3. But as known, each Si atom only has four outmost electrons, which means each $\mathrm{Si}$ atom can only form four bonds at most at stable status. And the positions cycled in Fig.3(b) already have formed four bonds, thus they are not preferred in chemical reaction. Instead, the atoms at above layer as shown in Fig.4(b) still have spare electron for bonding, thus this position is selected.

By calculation, spatial positions of surface atoms and atomic forces of all atoms have changed obviously after reaction. These issues will be discussed further in other paper to reveal formation mechanism of ultra-smooth surface in details. Here only the system energy is focuses on as mentioned ahead. The results are listed in Table 1. The binding energy of convex model can be calculated as

$$
\Delta E_{\text {convex }}=E_{\text {convex }+F}-\left(E_{\text {convex }}+E_{F}\right) \approx-347.7 \mathrm{eV}
$$

Similarly, the binding energy of concave model is $-347.5 \mathrm{eV}$. For one bonding with single $\mathrm{F}$ atom, binding energy of convex model is $0.2 \mathrm{eV}$ higher than concave model, which is thought to be distinct difference since the highest energy provided by one single fluorine atom is only $1 \mathrm{eV}$. For actual macroscale surface with millions of atoms, the difference value will be much bigger, because much more atoms will participate in reaction and the reaction process is much more complex than simulation. But as the computation load is too high to construct a huge model to simulate actual condition, especially for quantum chemistry tools, thus more detailed work will be undertaken on high capacity computers later. Nevertheless, for qualitative analysis, this simplified model is persuasive enough. And some other positions are also selected for simulation to testify the conclusion further. And all of them indicate that under the same condition, convex model has higher binding energy than 
concave model.

Table 1 Energy of models

$(\mathrm{eV})$

\begin{tabular}{c|c|c|c}
\hline & System energy before bonding & System energy after bonding & Binding Energy \\
\hline Convex model & -6468.8 & -7146.0 & -347.7 \\
\hline Concave model & -6469.3 & -7146.3 & -347.5 \\
\hline Fluorine Atom & \multicolumn{2}{|c}{-329.5} & \\
\hline
\end{tabular}

Besides, by comparing the models in Fig. 3 it is also found that with the same atom layers, convex structure has larger effective contacting area with fluorine atoms and weaker influence by local atomic configuration. And radical atoms contact convex structures above surface first when blown to surface, then with diffusion access to concave parts below surface while radical's density already decreases as the result of reacting with convex structures. Thus, convex topography is preferred to react. And due to higher reaction probability, convex topography can be removed faster than concave topography from general statistic view. So as machining progresses, surface goes flattened gradually.

\subsection{Experiments}

To testify theoretical simulation result, machining experiments on a 3-inch single crystal silicon wafer with lattice direction (100) have been performed. Experimental conditions are listed in the following table. Since the chamber has a specially designed ventilation pipe which keeps the gas flowing naturally to outside, the pressure in the chamber can be maintained at atmospheric pressure during operation.

Table 2 Experimental conditions

\begin{tabular}{l|c|c|c}
\hline Items & RF Power & $\mathrm{CF}_{4} / \mathrm{He}$ Ratio & Gap Distance \\
\hline Values & $400 \mathrm{~W}$ & $0.2 \%$ & $2 \mathrm{~mm}$ \\
\hline
\end{tabular}

According to theoretical analysis, convex topography should be removed faster than concave topography. Thus in experiments, the average roughness $\left(\mathrm{R}_{\mathrm{a}}\right)$, average maximum height $\left(R_{\mathrm{pm}}\right)$ and average maximum depth $\left(\mathrm{R}_{\mathrm{vm}}\right)$ were measured at $0 \mathrm{~s}, 40 \mathrm{~s}, 80 \mathrm{~s}$ and $120 \mathrm{~s}$ to be compared. The results are shown in Fig.5, from which it is clear that the average surface roughness decreases gradually as machining progresses, which indicates the surface topography goes smoother. The final roughness reaches below Ra $0.5 \mathrm{~nm}$ from original Ra1.179nm. And before machining, the surface is relatively rough with an average depth of $5.125 \mathrm{~nm}$ between highest and lowest points. And with reaction going, the difference becomes weaker and finally reaches $1.993 \mathrm{~nm}$. It proves the roughness caused by topography difference is reduced obviously. And respectively comparing reducing rate of convex topography and concave topography, it can be seen that in the same interval, $R_{\mathrm{pm}}$ is always reduced more than $\mathrm{R}_{\mathrm{vm}}$, as listed in Table 3 .

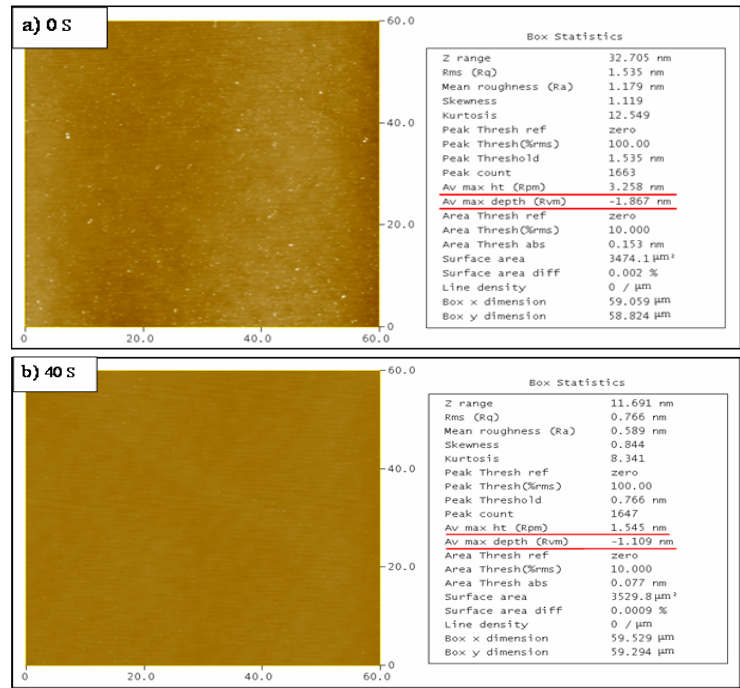



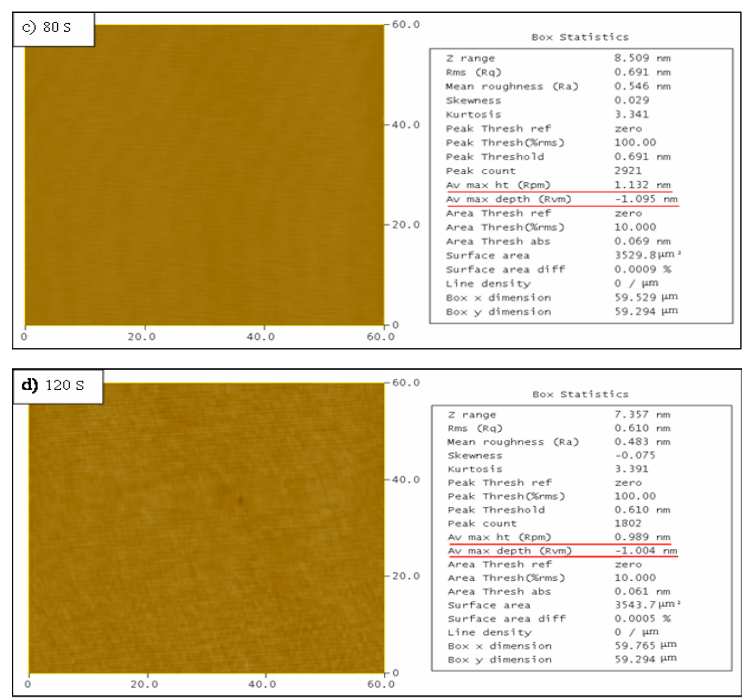

Fig. 5 Surface roughness detection images by AFM

Table 3 Reduction of $\mathrm{R}_{\mathrm{pm}}$ and $\mathrm{R}_{\mathrm{vm}}$ in different intervals $\quad$ (nm)

\begin{tabular}{c|c|c}
\hline \multirow{2}{*}{ Interval } & \multicolumn{2}{|c}{ Decreasing Amplitude } \\
\cline { 2 - 3 } & $\mathrm{R}_{\mathrm{pm}}$ & $\mathrm{R}_{\mathrm{vm}}$ \\
\hline $0 \mathrm{~s}-40 \mathrm{~s}$ & 1.713 & 0.758 \\
\hline $40 \mathrm{~s}-80 \mathrm{~s}$ & 0.413 & 0.014 \\
\hline $80 \mathrm{~s}-120 \mathrm{~s}$ & 0.143 & 0.091 \\
\hline
\end{tabular}

According well with the simulation, experiments prove that convex topography is removed faster than concave topography, thus the whole surface becomes smoother and smoother as machining progresses. And the reduction amplitude becomes smaller and smaller, which also indicates that the whole surface goes toward a new balance status.

\section{Improvement of Surface Mechanical Property}

Since APPP removes surface material by absolute chemical reaction, the shallow damage layer is usually eliminated effectively. Thus surface mechanical property can be improved with more regular lattice structure ${ }^{(9)(10)}$. For demonstration, experiments have been performed on 3-inch single crystal silicon wafer under the conditions listed in Table 4 for 5 minutes. And before operation, the wafer was tested by commercial nanomechanical test system (Hysitron, TriboIndenter) to measure the modulus of the original surface. Then after machining, the same region was detected again to testify the change of the mechanical property.

Table 4 Experimental conditions

\begin{tabular}{l|c|c|c}
\hline Items & RF Power & $\mathrm{CF}_{4} / \mathrm{He}$ Ratio & Gap Distance \\
\hline Values & $600 \mathrm{~W}$ & $0.2 \%$ & $5 \mathrm{~mm}$ \\
\hline
\end{tabular}

In Table 5, the tested reduced-modulus of the same surface before and after machining $\left(\mathrm{E}^{*}\right.$ and $\left.\mathrm{E}_{0}{ }^{*}\right)$ is listed. From the results, it is clear that after machining the reduced-modulus of the surface is much larger than that of the original surface. The later average value is more than two times larger than the former one. And the largest difference between the former and later values even reaches $28.21 \mathrm{GPa}$. The hardness of machined surface $\left(\mathrm{H}_{0}\right)$ is also testified to be larger than that of original surface $\left(\mathrm{H}_{0}{ }^{*}\right)$. Thus, it can be concluded that the APPP process is capable of improving the rigidity of surface.

Furthermore, the relative value of residual stress of the original surface $\left(\sigma_{R}\right)$ can be deduced based on above detected parameters of machined surface. Carlsson and Larsson method is adopted ${ }^{(11)}$, which is not redundantly explained in detail here. The calculation 
Table 5 Test results of the nanomechanical test system

\begin{tabular}{c|c|c|c|c|c|c}
\hline & 1 & 2 & 3 & 4 & 5 & Ave \\
\hline Before machining & & & & & & \\
$\mathrm{E}^{*}$ (GPa) & 14.37 & 13.63 & 14.15 & 16.18 & 14.91 & 14.65 \\
$\mathrm{H}^{*}$ (GPa) & 4.64 & 4.16 & 3.69 & 3.66 & 4.74 & 4.18 \\
\hline After machining & & & & & & \\
E $_{0}{ }^{*}$ (GPa) & 29.24 & 41.84 & 36.74 & 35.07 & 37.59 & 36.10 \\
H$_{0}{ }^{*}$ (GPa) & 4.63 & 6.14 & 5.14 & 8.11 & 7.55 & 6.31 \\
\hline
\end{tabular}

results are shown in Table 6. It is clear that the average value of reduced residual stress is $7.64 \mathrm{GPa}$. So APPP performs well on the elimination of surface stress or the removal of deformation layer.

Table 6 Calculation results of residual stress $\sigma_{R}$

\begin{tabular}{|c|c|c|c|c|c|c|}
\hline Point number & 1 & 2 & 3 & 4 & 5 & Ave \\
\hline$\sigma_{\mathrm{R}} \quad(\mathrm{GPa})$ & 7.87 & 8.73 & 8.11 & 6.17 & 7.31 & 7.64 \\
\hline
\end{tabular}

\section{Improvement of Surface Element Composition}

The surface element composition was detected by XPS (X-ray photoelectron spectroscopy, PHI 5700 ESCA system; Physical Electronics, USA). Here only the silicon element is discussed, although the fluorine, oxygen and carbon element were also detected. From Fig.6 it is clear that before machining, silicon exists on the surface in two forms: $\mathrm{Si}$ and $\mathrm{SiO}_{2}$. The left peak in Fig.6(a) stands for $\mathrm{Si}$ while the right lower peak is $\mathrm{SiO}_{2}$. Obviously, only a small part of silicon element is oxidized since the height of the peak correspond to component proportion. And the appearance of oxide is rational because silicon tends to be oxidized under air to form more stable compound. And from Fig.6(b) it can be found after machining, $\mathrm{SiO}_{2}$ was eliminated thoroughly which proves more purified element composition. And the later detection didn't find oxide mainly depends on the seal isolation of machined sample from outside environment. Besides simple substance, other forms of silicon element are not found on machined surface, so APPP does not introduce new contamination.

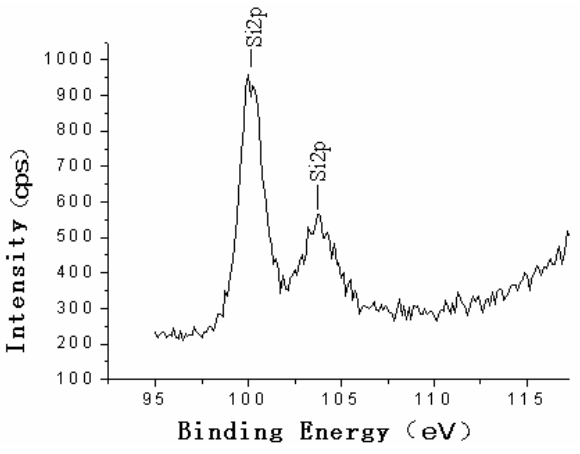

(a) before machining

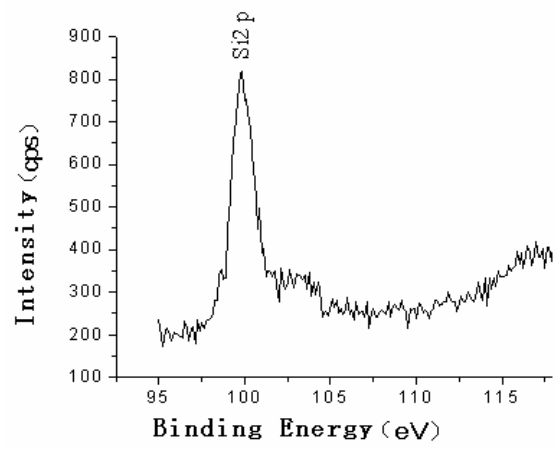

(b) after machining

Fig. 6 Si element composition detected by XPS

The general status of whole surface is detected and analyzed by X-ray diffraction (X'Pert, Philips) before and after machining. Diffraction mode is $\theta \sim 2 \theta$ scanning, and the result is shown in Fig.7. There are two peaks with the same axis at $69.132^{\circ}$ which stands for silicon element. And the half peak width of machined surface is obviously narrower than that of original surface, while the intensity of machined surface is much higher than that of original surface. According to analysis theory of X-ray diffraction, the ideal peak of element should have high intensity and small half peak width, so it is clear that the machined surface is more close to perfect single crystal silicon status. 


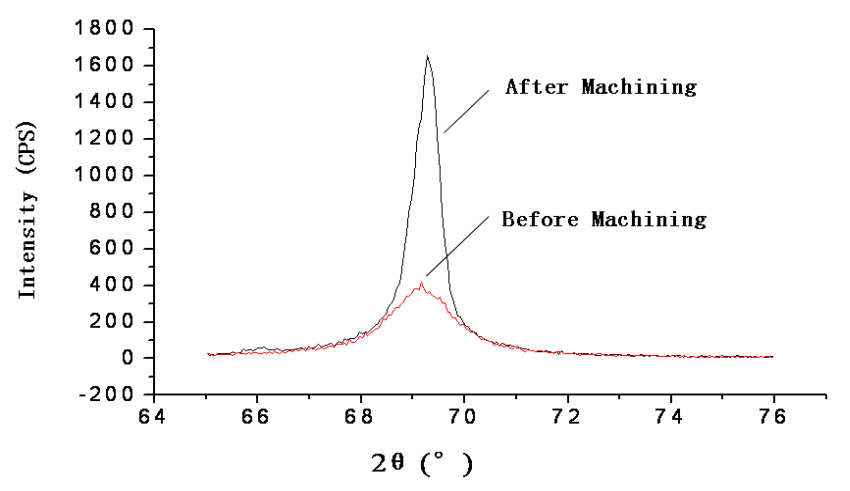

Fig. 7 X-ray diffraction test results

\section{Conclusions}

Atmospheric pressure plasma polishing has been proved to be valid in improving surface roughness to form ultra-smooth surface, reducing residual stress to enhance surface mechanical properties, ameliorate chemical composition for better performance. And it does not introduce new surface damage or contamination. Thus, it is capable of supporting manufacturing high quality optical surfaces for hard brittle materials.

\section{Acknowledgement}

This project was supported by the Improving Foundation of Shenzhen Digital Manufacturing Lab (AZ24412006). We also thank Professor Yuan Fulong of Heilongjiang University for supporting and instructing on quantum chemistry simulation.

\section{References}

(1)Zhang, J.F., Wang, B. and Dong, S., A New Development of Ultra-smooth Surface Machining Technology, Optical Technique, Vol. 33, No. S1(2007), pp. 150-154.

(2)Xie, H.D., Wang, X.D. and Shen, G.Q., Superpolishing of Crystals, Journal of Synthetic Crystals, Vol. 33, No. 6(2004), pp. 1035-1040.

(3)Chen, Y., Chen, J.Q. and Chen, Z.G., Ultra-Smooth Surface Polishing Techniques, Journal of Jiangsu University (National Science Edition), Vol. 24, No. 5(2003), pp. 55-59.

(4)Li, K. and Liao, T.W., Surface/Subsurface Damage and Fracture Strength of Ground Ceramics, J. Mat. Proc.Tech., Vol. 57, No. 3-4(1996), pp. 207-220.

(5)Li, B.G., Xiong, C.Y., Li, C.G. and Zhang, Q.R., Machining Technology of Super Smooth Surface, Manufacturing Technology \& Machine Tool, No. 6(2006), pp. 60-66.

(6)Zhang, J.F., Wang, B. and Dong, S., Process Monitoring and Analysis in the Atmospheric Pressure Plasma Polishing (APPP) Method, Proc.of SPIE, Vol. 6722(2007), pp.67220O.

(7)Wang, B., Zhang, J.F. and Dong, S., The Application of the Atomic Emission Spectroscopy Analysis in the Atmospheric Pressure Plasma Polishing Process Study, Spectroscopy and Spectral Analysis, Vol. 28, No. 7(2008), pp. 1641-1644.

(8)Zhang, J.F., Wang, B. and Dong, S., Design of the Atmospheric Pressure Plasma Polishing System for the Machining of Ultra-Smooth Surfaces, Nanotechnology and Precision engineering, Vol. 6, No. 3(2008), pp. 222-226.

(9)Zhang, J.F., Wang, B. and Dong, S., The Application of the Atmospheric Pressure Plasma Polishing (APPP) Method in the Machining of Silicon Ultra-smooth Surfaces, Optics and Precision Engineering, Vol. 15, No. 11(2007), pp. 1759-1755.

(10)Zhang, J.F., Wang, B. and Dong, S., Analysis of Factors Impacting Atmospheric Pressure Plasma Polishing, Int. J. Precis. Eng. Manuf., Vol. 9, No. 2(2008), pp. 39-43.

(11)Carlsson, S. and Larsson, P.L., On the Determination of Residual Stress and Strain Fields by Sharpindentation Testing.: Part II: Experimental Investigation, J. Mater. Res., Vol. 49, No. 12(2001), pp. 2193-2203. 